\title{
NLTE strontium abundance in a sample of extremely metal poor stars and the Sr/Ba ratio in the early Galaxy
}

\author{
S. M. Andrievsky ${ }^{1,2}$, F. Spite ${ }^{1}$, S. A. Korotin ${ }^{2}$, P. François ${ }^{1}$, M. Spite ${ }^{1}$, P. Bonifacio ${ }^{1,3}$, R. Cayrel $^{1}$, and V. Hill ${ }^{1}$ \\ 1 GEPI, Observatoire de Paris-Meudon, CNRS, Université Paris Diderot, 92125 Meudon Cedex, France \\ 2 Department of Astronomy and Astronomical Observatory, Odessa National University, and Isaac Newton Institute of Chile Odessa \\ branch, Shevchenko Park, 65014 Odessa, Ukraine \\ e-mail: scan@deneb1.odessa.ua \\ ${ }^{3}$ Istituto Nazionale di Astrofisica - Osservatorio Astronomico di Trieste, via Tiepolo 11, 34143 Trieste, Italy
}

Received 26 January 2011 / Accepted 26 February 2011

\section{ABSTRACT}

\begin{abstract}
Context. Heavy element abundances in extremely metal-poor stars provide strong constraints on the processes of forming these elements in the first stars.

Aims. We attempt to determine precise abundances of strontium in a homogeneous sample of extremely metal-poor stars.

Methods. The abundance of strontium in 54 very or extremely metal-poor stars, was redetermined by abandoning the local thermodynamic equilibrium (LTE) hypothesis, and fitting non-LTE (NLTE) profiles to the observed spectral lines. The corrected $\mathrm{Sr}$ abundances and previously obtained NLTE Ba abundances are compared to the predictions of several hypothetical formation processes for the lighter neutron-capture elements.

Results. Our NLTE abundances confirm the previously determined huge scatter of the strontium abundance in low metallicity stars. This scatter is also found (and is even larger) at very low metallicities (i.e. early in the chemical evolution). The Sr abundance in the extremely metal-poor (EMP) stars is compatible with the main r-process involved in other processes (or by variations of the r-process), as we briefly discuss.
\end{abstract}

Key words. Galaxy: abundances - Galaxy: halo - Galaxy: evolution - stars: abundances - supernovae: general

\section{Introduction}

Until recently, our knowledge of the properties of the first stellar generations of the Milky Way was quite restricted. Large telescopes and efficient detectors have made it possible to determine the abundances of many chemical elements of very primitive (very metal-poor) stars, constraining the theories of nucleosynthesis in the first stars.

The analysis of extremely metal-poor stars (EMP) provide important information about the abundances of the elements produced by the first stars, and about the corresponding formation processes. The abundances of the heavy elements $(Z>30)$ are particularly interesting. Several processes have been proposed to explain their production, especially two important neutroncapture processes: the rapid process (r-process) and the slow process (s-process). Each of these processes is divided into two sub-processes ("main" and "weak").

Massive stars with a very short lifetime end their lives as core-collapse SN II, forming neutron stars, and it had been assumed that the primitive massive stars provide a promising site for the early production of the neutron-capture elements by the r-process (see e.g. Cowan \& Thielemann 2004; Farouqi et al. 2009). The hypothesis was thought to be consistent with these massive stars being the primary early producers of light elements (called $\alpha$ elements), this production declining at later phases of the chemical evolution of the Galaxy, as (roughly) for the r-process elements.

However, developments in nucleosynthesis models (e.g. Hüdepold et al. 2010; Fisher et al. 2010; and Roberts et al. 2010) show that the classical neutrino-driven wind of core-collapse $\mathrm{SNe}$ is unable to produce the r-elements: they could instead be produced independently in other ways as proposed by Wanajo et al. (2010).

The neutron-capture elements may also be produced by a "slow" neutron flux, but in the "main" s-process the production takes place in intermediate-mass AGB stars, which have a long evolution time, that is inconsistent with an early production. Some early production could take place by means of the "weak" s-process, in massive primitive stars although the lack of seed nuclei places this idea in doubt.

In the Solar System, the global abundances of the heavy elements are the result of a late production by the main s-process in addition to an early production by the r-process, and it has been possible to distinguish the fraction produced by each process.

Cowan \& Sneden (2006) demonstrated that the abundances of heavy (56 $<Z<70)$ elements, display nearly identical abundance patterns in the r-fraction of the abundances of the Solar System, and in 6 r-rich EMP stars (some more r-rich EMP stars have been added recently to the list). As a result, the main r-process has been proposed to be universal. With larger samples of analyzed stars, the whole picture appeared more complex. It is therefore quite important to determine the precise abundances of heavy elements in EMP stars. Since the electron concentration in the atmospheric plasma of these stars is substantially lower, significant deviations from LTE in these atmospheres are expected. Thus, reliable abundances of these stars can be obtained only by means of a NLTE analysis. As noted by Mashonkina \& Gehren (2001), the NLTE correction can be significant, and 
clearly differs from one star to another. We therefore decided to reanalyze the sample of the EMP stars previously studied by François et al. (2007) and Bonifacio et al. (2009) in the framework of the ESO large program "First stars, first nucleosynthesis". For this work, we determined for these stars the abundance of Sr based on NLTE computations of the line profiles. We combine these abundances with previous NLTE determinations of $\mathrm{Ba}$ abundances (see Andrievsky et al. 2009) to study the evolution of the $[\mathrm{Sr} / \mathrm{Ba}]$ ratio in the early phases of the chemical evolution of the Galaxy.

\section{Sample of stars and their parameters}

The spectra of the stars in this sample were presented in detail in Cayrel et al. (2004) and Bonifacio et al. (2007, 2009). About fifty stars (35 giants and 18 turnoff stars), most of them with $[\mathrm{Fe} / \mathrm{H}]<-3$, were observed with the high-resolution spectrograph UVES (Dekker et al. 2000) fed by the ESO-VLT. The resolving power in the blue is $R \approx 45000$. The spectra were reduced using the UVES reduction tool within MIDAS (Ballester et al. 2000). The signal-to-noise ratio $(S / N)$ in the region of the blue strontium lines is typically $\sim 120$ per pixel with there being an average of 5 pixels per resolution element. The sample includes a few well-known typical stars for comparison.

The fundamental parameters $\left(T_{\text {eff }}, \log g,[\mathrm{Fe} / \mathrm{H}]\right)$ of the stars were derived by Cayrel et al. (2004) for the giants and Bonifacio et al. (2007) for the turn-off stars. The temperature of the giants is deduced from the colour by adopting the calibration of Alonso et al. (1996), and the temperature of the turn-off stars is deduced from the wings of the $\mathrm{H} \alpha$ line. The gravities are derived from the ionisation equilibrium of iron (in LTE approximation), and could be affected by NLTE effects. These parameters are repeated in Table 1.

Briefly, MARCS model atmospheres have been used, but, for convenience, some parts of the computations have used ATLAS9 (Kurucz 1993, 2005) model atmospheres with the overshooting option. It has been shown (Castelli et al. 1997) that for EMP stars, non-overshooting models are more appropriate. Nonovershooting Kurucz models have been shown to provide abundances very similar (within $0.05 \mathrm{dex}$ ) to those of the MARCS models used by Cayrel et al. (2004) and Bonifacio et al. (2009).

Table 1 provides the adopted parameters and the $\mathrm{Sr}$ abundances. In addition we present the $\mathrm{Ba}$ abundances determined in the same way in Andrievsky et al. (2009) but using Kurucz models with the overshooting option (Kurucz 1993, 2005). As a consequence, these previous NLTE Ba abundances are shifted (Table 1 ) by -0.03 dex for the turn-off stars, and -0.05 dex for the giants.

Three new giant stars were added to the original sample, their parameters and NLTE abundances (including barium) being determined in the same way; they are listed at the end of Table 1. In the last column, we indicate the number of lines of $\mathrm{Sr}$ II measured.

Two carbon-rich stars (CEMPs) are included in the sample (they are designated by an asterisk in the first column of Table 1): CS 22949-037 (Sr-rich) and CS 22892-052 (a useful typical example of an extremely r-rich star). Among the turn-off stars, CS $29527-015$ is a spectroscopic binary.

\section{Atomic model and calculations}

\subsection{The strontium atomic model}

A NLTE analysis of the Sr II spectrum has been the subject of only a few papers. A first application of the NLTE calculations

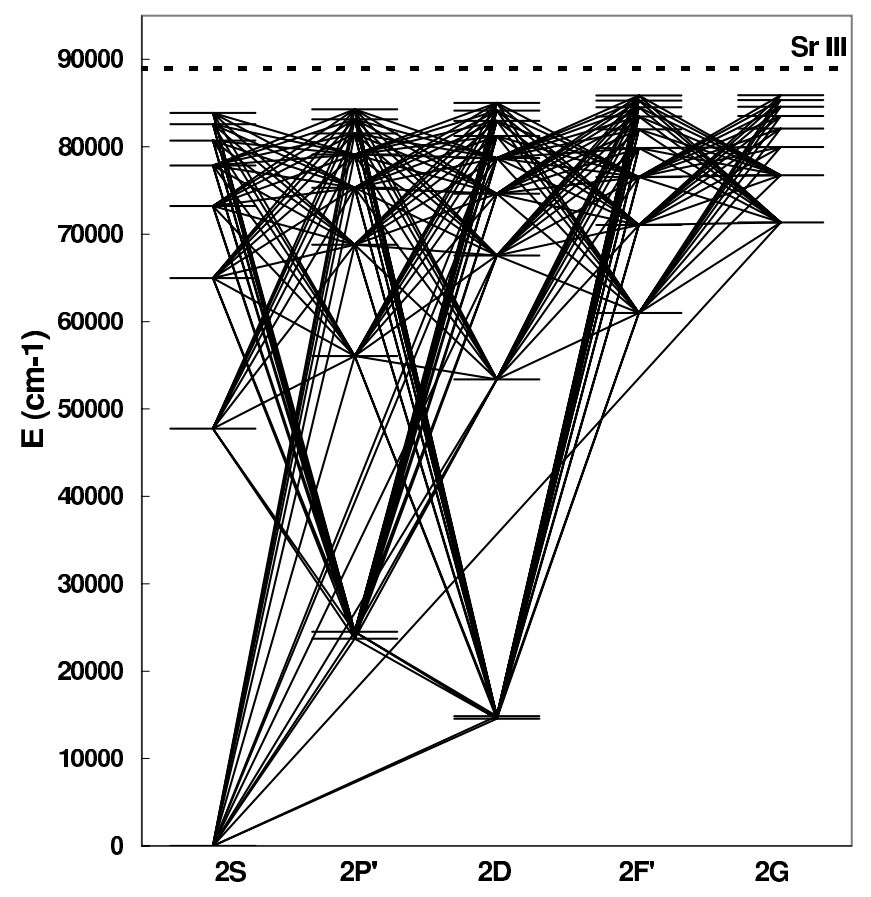

Fig. 1. The Grotrian diagram for $\mathrm{Sr}$ II atom.

for Ap-Bp stars was made by Borsenberger et al. (1981), who used a six-level (plus continuum) atomic model of Sr II. Belyakova \& Mashonkina (1997) considered the statistical equilibrium of Sr II in atmospheres of F-G stars of different luminosity classes with metallicity ranging from zero to -3 . They used a model atom consisting of 40 levels of $\mathrm{Sr}$ II and the ground level of the Sr III ion, and this model was applied to derive NLTE corrections. After this, Mashonkina \& Gehren (2001) determined NLTE abundances in 63 cool stars with metallicity ranging from +0.25 to -2.20 . Mashonkina et al. (2008) published results for the NLTE abundances of some heavy elements (including strontium) in four very metal-poor (VMP) stars with metallicity in the range from -2.1 to -2.7 .

Our strontium atomic model is quite similar to that first proposed by Belyakova \& Mashonkina (1997). The Grotrian diagram is given in Fig. 1. The model consists of 44 levels of $\mathrm{Sr}$ II with $n<13$ and $l<6$, as well as the ground level of Sr III (no excited levels of this ion were included, since they have very high excitation potentials). For the two important terms $4 d^{2} \mathrm{D}$ and $5 \mathrm{p}^{2} \mathrm{P}^{\circ}$, the fine structure was taken into account. For the other levels, it was ignored.

Since the ionization potential of the neutral strontium is only $5.7 \mathrm{eV}$, even in the atmospheres of cool stars it exists almost only in the form of Sr II. Therefore, the 24 levels of Sr I were included in the model only for the particle number conservation.

\subsection{The calculations}

For all considered levels, the excitation energies were taken from Lindgard \& Nielsen (1977). Oscillators strengths for 72 boundbound transitions were selected from three papers: Wiese \& Martin (1980), Warner (1968), and Goldsmith \& Boxman (1981). For the remaining 258 transitions, we used oscillator strengths from Lingard \& Nielsen (1977).

Radiative photoionization rates for the $s, \mathrm{p}$, and d levels are based on the photoionization cross-sections calculated with the 
Table 1. Parameters of program stars and strontium abundance.

\begin{tabular}{|c|c|c|c|c|c|c|c|c|c|}
\hline $\begin{array}{l}\text { Star } \\
\text { turnoff stars }\end{array}$ & $T_{\text {eff }}, \mathrm{K}$ & $\log g$ & $V_{\mathrm{t}}, \mathrm{km} \mathrm{s}^{-1}$ & {$[\mathrm{Fe} / \mathrm{H}]$} & $\epsilon(\mathrm{Sr})$ & {$[\mathrm{Sr} / \mathrm{H}]$} & {$[\mathrm{Sr} / \mathrm{Fe}]$} & {$[\mathrm{Ba} / \mathrm{H}]$} & Rem \\
\hline BS 16023-046 & 6360 & 4.5 & 1.4 & -2.97 & -0.08 & -3.00 & -0.03 & - & 2 \\
\hline BS 16968-061 & 6040 & 3.8 & 1.5 & -3.05 & -0.46 & -3.38 & -0.33 & - & 2 \\
\hline BS 17570-063 & 6240 & 4.8 & 0.5 & -2.92 & +0.10 & -2.82 & +0.10 & -2.97 & 2 \\
\hline CS 22177-009 & 6260 & 4.5 & 1.2 & -3.10 & -0.09 & -3.01 & +0.09 & - & 2 \\
\hline CS 22888-031 & 6150 & 5.0 & 0.5 & -3.30 & -0.13 & -3.05 & +0.25 & - & 2 \\
\hline CS 22948-093 & 6360 & 4.3 & 1.2 & -3.30 & -0.20 & -3.12 & +0.18 & -2.97 & 2 \\
\hline CS 22965-054 & 6090 & 3.8 & 1.4 & -3.04 & +0.20 & -2.72 & +0.32 & - & 2 \\
\hline CS 22966-011 & 6200 & 4.8 & 1.1 & -3.07 & +0.07 & -2.85 & +0.22 & -2.87 & 2 \\
\hline CS 29499-060 & 6320 & 4.0 & 1.5 & -2.70 & -0.15 & -3.07 & -0.37 & - & 2 \\
\hline CS 29506-007 & 6270 & 4.0 & 1.7 & -2.91 & +0.31 & -2.61 & +0.30 & -2.37 & 2 \\
\hline CS 29506-090 & 6300 & 4.3 & 1.4 & -2.83 & +0.54 & -2.38 & +0.45 & -2.77 & 2 \\
\hline CS 29518-043 & 6430 & 4.3 & 1.3 & -3.20 & +0.00 & -2.92 & +0.28 & - & 2 \\
\hline CS 29527-015 & 6240 & 4.0 & 1.6 & -3.55 & -0.06 & -2.98 & +0.57 & - & 2 \\
\hline CS 30301-024 & 6330 & 4.0 & 1.6 & -2.75 & +0.27 & -2.65 & +0.10 & -2.67 & 2 \\
\hline CS 30339-069 & 6240 & 4.0 & 1.3 & -3.08 & -0.11 & -3.03 & +0.05 & - & 2 \\
\hline CS 31061-032 & 6410 & 4.3 & 1.4 & -2.58 & +0.55 & -2.37 & +0.21 & - & 2 \\
\hline \multicolumn{10}{|l|}{ giants } \\
\hline HD 2796 & 4950 & 1.5 & 2.1 & -2.47 & +0.57 & -2.35 & +0.12 & -2.68 & 4 \\
\hline HD 122563 & 4600 & 1.1 & 2.0 & -2.82 & +0.10 & -2.82 & +0.00 & -3.67 & 5 \\
\hline HD 186478 & 4700 & 1.3 & 2.0 & -2.59 & +0.57 & -2.35 & +0.24 & -2.67 & 4 \\
\hline $\mathrm{BD}+17^{\circ} 3248$ & 5250 & 1.4 & 1.5 & -2.07 & +1.17 & -1.75 & +0.32 & -1.74 & 2 \\
\hline $\mathrm{BD}-18^{\circ} 5550$ & 4750 & 1.4 & 1.8 & -3.06 & -0.93 & -3.85 & -0.79 & -3.67 & 3 \\
\hline $\mathrm{CD}-38^{\circ} 245$ & 4800 & 1.5 & 2.2 & -4.19 & -1.75 & -4.67 & -0.48 & -4.72 & 2 \\
\hline BS 16467-062 & 5200 & 2.5 & 1.6 & -3.77 & -2.30 & -5.22 & -1.45 & - & 2 \\
\hline BS 16477-003 & 4900 & 1.7 & 1.8 & -3.36 & -0.40 & -3.32 & +0.04 & -3.62 & 2 \\
\hline BS 17569-049 & 4700 & 1.2 & 1.9 & -2.88 & +0.45 & -2.47 & +0.41 & -2.72 & 4 \\
\hline CS 22169-035 & 4700 & 1.2 & 2.2 & -3.04 & -0.33 & -3.25 & -0.21 & -4.17 & 2 \\
\hline CS 22172-002 & 4800 & 1.3 & 2.2 & -3.86 & -2.03 & -4.95 & -1.09 & -4.82 & 2 \\
\hline CS 22186-025 & 4900 & 1.5 & 2.0 & -3.00 & -0.18 & -3.10 & -0.10 & -2.94 & 2 \\
\hline CS 22189-009 & 4900 & 1.7 & 1.9 & -3.49 & -1.28 & -4.20 & -0.71 & -4.62 & 2 \\
\hline CS 22873-055 & 4550 & 0.7 & 2.2 & -2.99 & -0.40 & -3.32 & -0.33 & -3.37 & 2 \\
\hline CS 22873-166 & 4550 & 0.9 & 2.1 & -2.97 & +0.27 & -2.65 & +0.32 & -3.54 & 3 \\
\hline CS 22878-101 & 4800 & 1.3 & 2.0 & -3.25 & -0.58 & -3.50 & -0.25 & -3.57 & 2 \\
\hline CS 22885-096 & 5050 & 2.6 & 1.8 & -3.78 & -2.00 & -4.92 & -1.14 & -4.72 & 2 \\
\hline CS 22891-209 & 4700 & 1.0 & 2.1 & -3.29 & -0.23 & -3.15 & +0.14 & -3.65 & 2 \\
\hline CS 22892-052* & 4850 & 1.6 & 1.9 & -3.03 & +0.47 & -2.45 & +0.58 & -2.25 & 4 \\
\hline CS 22896-154 & 5250 & 2.7 & 1.2 & -2.69 & +0.67 & -2.25 & +0.44 & -2.35 & 3 \\
\hline CS 22897-008 & 4900 & 1.7 & 2.0 & -3.41 & -0.08 & -3.00 & +0.41 & -4.37 & 2 \\
\hline CS 22948-066 & 5100 & 1.8 & 2.0 & -3.14 & -0.68 & -3.60 & -0.46 & -3.97 & 2 \\
\hline CS 22949-037* & 4900 & 1.5 & 1.8 & -3.97 & -0.88 & -3.80 & +0.17 & -4.47 & 2 \\
\hline CS 22952-015 & 4800 & 1.3 & 2.1 & -3.43 & -1.33 & -4.25 & -0.82 & -4.57 & 2 \\
\hline CS 22953-003 & 5100 & 2.3 & 1.7 & -2.84 & +0.30 & -2.62 & +0.22 & -2.45 & 2 \\
\hline CS 22956-050 & 4900 & 1.7 & 1.8 & -3.33 & -0.80 & -3.72 & -0.39 & -3.95 & 2 \\
\hline CS 22966-057 & 5300 & 2.2 & 1.4 & -2.62 & +0.12 & -2.80 & -0.18 & -2.95 & 2 \\
\hline CS 22968-014 & 4850 & 1.7 & 1.9 & -3.56 & -2.17 & -5.09 & -1.53 & - & 2 \\
\hline CS 29491-053 & 4700 & 1.3 & 2.0 & -3.04 & -0.33 & -3.25 & -0.21 & -3.74 & 2 \\
\hline CS 29495-041 & 4800 & 1.5 & 1.8 & -2.82 & -0.13 & -3.05 & -0.23 & -3.28 & 2 \\
\hline CS 29502-042 & 5100 & 2.5 & 1.5 & -3.19 & -1.98 & -4.90 & -1.71 & -4.62 & 2 \\
\hline CS 29516-024 & 4650 & 1.2 & 1.7 & -3.06 & -0.63 & -3.55 & -0.49 & -3.67 & 2 \\
\hline CS 29518-051 & 5200 & 2.6 & 1.4 & -2.69 & +0.45 & -2.47 & +0.22 & -2.82 & 2 \\
\hline CS 30325-094 & 4950 & 2.0 & 1.5 & -3.30 & -2.37 & -5.29 & -1.99 & - & 2 \\
\hline $\begin{array}{l}\text { CS 31082-001 } \\
\text { additional giants }\end{array}$ & 4825 & 1.5 & 1.8 & -2.91 & +0.52 & -2.40 & +0.51 & -2.17 & 4 \\
\hline CS 22891-200 & 4700 & 1.3 & 2.0 & -3.65 & -1.75 & -4.67 & -1.02 & -4.28 & 2 \\
\hline CS 22949-048 & 4800 & 1.5 & 2.0 & -3.25 & -1.39 & -4.31 & -1.06 & -4.33 & 2 \\
\hline CS 22950-046 & 4650 & 1.0 & 2.2 & -3.56 & -1.12 & -4.04 & -0.48 & -4.49 & 2 \\
\hline
\end{tabular}

Notes. The Ba abundance has been corrected for overshooting.

quantum defect method and the corresponding tables of Peach (1967). For $\mathrm{f}$ and g levels, we used the hydrogen-like approximation (Lang 1998).

Collisional rates for the transitions between the ground level $5 s^{2} \mathrm{~S}$ and $5 \mathrm{p}^{2} \mathrm{P}^{\circ}, 5 \mathrm{~d}^{2} \mathrm{D}$, and $6 \mathrm{~s}^{2} \mathrm{~S}$ levels were estimated with the help of the corresponding formula from Sobelman et al. (1981). For the remaining levels, the excitation rates by electron collisions were approximated using the formula of van Regemorter (1962). Collisional excitations for the forbidden transitions were taken into account using the semi-empirical formula of 
Allen (1973) with a factor of 1 . Collisional ionization caused by electrons was described using the Drawin (1961) formula. Inelastic collisions with hydrogen atoms were taken into account with the formula of Steenbock \& Holweger (1992) with a scaling coefficient 0.01 . This coefficient was derived from the fitting of the Sr II line profiles in the solar spectrum (Kurucz et al. 1984).

The NLTE strontium abundance was determined with a modified MULTI code (Carlsson 1986). Modifications are described in Korotin et al. (1999). Since we use Kurucz's atmosphere models calculated with ATLAS 9 (Kurucz 1993, 2005), the necessary background opacities for MULTI are also taken from ATLAS 9. In the modified version, the mean intensities used to obtain the radiative photoionization rates, are calculated for the set of frequencies at each atmospheric layer, and then stored in a separate block, where they can be interpolated. This enables us to take account of the absorption in a great number of lines, especially in the UV region, which is important for the accuracy of the photoionization rate calculations.

After the combined solution of the statistical equilibrium and radiative transfer equations, we derived the populations of the $5 \mathrm{~s}^{2} \mathrm{~S}, 4 \mathrm{~d}^{2} \mathrm{D}, 5 \mathrm{p}^{2} \mathrm{P}^{\circ}$, and $6 \mathrm{~s}^{2} \mathrm{~S}$ levels allowing us to reproduce the profiles of the lines of interest, provided that the broadening parameters were known. Those parameters were taken from the VALData-base ${ }^{1}$.

In the visual part of the stellar spectra and near-IR range, there are only a few detectable lines of Sr II, including the resonance lines $4077 \AA$, $4215 \AA$, and the subordinate lines $10036 \AA$, $10327 \AA$, and $10914 \AA$. We note that resonance lines are significantly blended with iron, chromium, as well as some molecular lines. The IR lines are not blended. The $10914 \AA$ line is unavailable in the UVES spectra, but the two other IR lines $10036 \AA$ and $10327 \AA$ are, even though in some EMP stars they are both detected at the noise level.

To compare the NLTE strontium line profiles to the observed spectrum in the visual part of the spectrum one needs to use a combination of the NLTE and LTE synthetic spectrum since the resonance $\mathrm{Sr}$ II lines are to some extent blended with other metallic lines. This was achieved using the updated code SYNTHV (Tsymbal 1996) which is designed for synthetic spectrum calculations in LTE. With this program, we calculated a synthetic spectrum for particular wavelength ranges including the $\mathrm{Sr}$ II lines of interest taking into account all the lines from each region listed in the VALData-base. For the strontium lines, the corresponding $b$-factors (factors of deviation from LTE level populations) that had been calculated in MULTI were included in SYNTHV, where they were used in calculating the strontium line source function (in particular, the first plot in Fig. 2 gives an example of this procedure application).

\subsection{Test calculations}

All the above listed input data and our strontium atomic model were checked using several test calculations. For this, we used high-resolution spectra of some stars with well known parameters.

First of all, we calculated profiles for the six Sr II lines in the solar spectrum (visual and infrared range, Kurucz's et al. 1984, solar flux spectrum) using the Kurucz (1996) solar atmosphere model with microturbulence of $1 \mathrm{~km} \mathrm{~s}^{-1}$. The strontium lines parameters are given in Table 2.

The $\mathrm{Sr}$ II lines in the solar spectrum are closely reproduced by our calculations (see Fig. 2) with strontium abundance $(\mathrm{Sr} / \mathrm{H})=$

\footnotetext{
${ }^{1}$ http://ams.astro.univie.ac.at/vald/
}

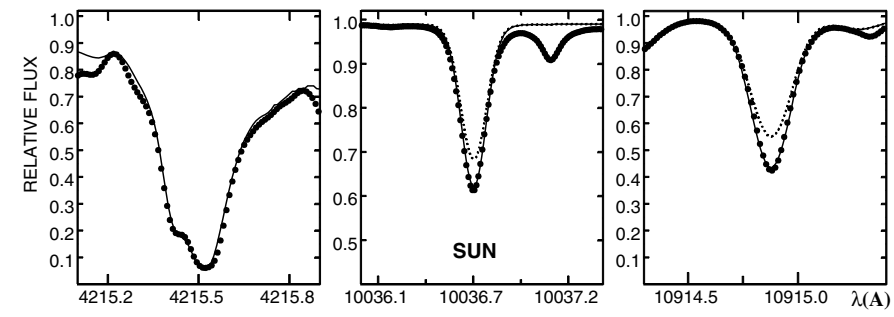

Fig. 2. Profile fitting of the $\mathrm{Sr}$ II lines in the solar spectrum. Observed spectrum - dots, NLTE profiles - smooth line, LTE profiles - dotted line.

Table 2. Parameters of the Sr II lines.

\begin{tabular}{lccc}
\hline \hline$\lambda, \AA$ & $f$ & $\log \gamma_{\mathrm{rad}}$ & $\log \gamma_{\mathrm{VW}}$ \\
\hline 4077.7090 & $7.063 \mathrm{e}-1$ & 8.130 & -7.70 \\
4161.7920 & $1.945 \mathrm{e}-1$ & 8.110 & -7.60 \\
4215.5190 & $3.303 \mathrm{e}-1$ & 8.100 & -7.70 \\
4305.4430 & $1.811 \mathrm{e}-1$ & 8.080 & -7.60 \\
10036.6530 & $1.219 \mathrm{e}-2$ & 7.340 & -7.63 \\
10327.3110 & $7.393 \mathrm{e}-2$ & 7.320 & -7.63 \\
10914.8870 & $5.754 \mathrm{e}-2$ & 7.270 & -7.63 \\
\hline
\end{tabular}

Table 3. Fundamental parameters of the reference stars and their NLTE Sr abundance.

\begin{tabular}{lccccc}
\hline \hline Star & $T_{\text {eff }}, \mathrm{K}$ & $\log g$ & $V_{\mathrm{t}}, \mathrm{km} \mathrm{s}^{-1}$ & {$[\mathrm{Fe} / \mathrm{H}]$} & $(\mathrm{Sr} / \mathrm{H})$ \\
\hline Arcturus & 4300 & 1.5 & 1.7 & -0.33 & 2.26 \\
Procyon & 6500 & 4.0 & 1.8 & +0.00 & 3.12 \\
HD 1581 & 6000 & 3.9 & 1.0 & -0.22 & 2.69 \\
HD122563 & 4600 & 1.1 & 2.0 & -2.82 & 0.10 \\
\hline
\end{tabular}

2.92 (as recommended by Asplund et al. 2005). For the sake of completeness, we also provide pure LTE profiles for all investigated lines.

We also compared the calculated $\mathrm{Sr}$ II line profiles with those observed in the following well studied stars: Arcturus, Procyon, HD 1581, and the metal-deficient star HD 122563 (which is also in our list of program stars). For these stars, spectra are available from the UVES archive of the Paranal Observatory Project (Bagnulo et al. 2003). These spectra were obtained with $R=80000$ and have $S / N$ of more than 300. Since the third Sr II IR line $10914 \AA$ is unavailable in the UVES spectra (but is important for the elaborated strontium atomic model check), we used the Keck IR spectrum of HD 122563 previously analyzed in Smith et al. (2004). Adopted parameters of these stars are given in Table 3. The estimated NLTE strontium abundance of these stars is given in the last column of this table.

Figure 3 shows that the Sr II lines in HD 122563 that belong to the different multiplets are well reproduced with a single strontium abundance.

Very good agreement is obtained between the abundances derived from the lines in the infrared and visible domains for the stars HR 1581 and HD 122563. For HD 122563, the abundance of $\mathrm{Sr}$ (and $\mathrm{Ba}$ ) is also in very good agreement with the NLTE results of Mashonkina et al. (2008).

\section{The behaviour of the $b$-factors}

Figure 4 shows the behaviour of the $b$-factors for the solar atmosphere. As one can see, $b$-factors of the 5 p level are smaller than those for $5 \mathrm{~s}$ level over the whole atmosphere. At the same time, 

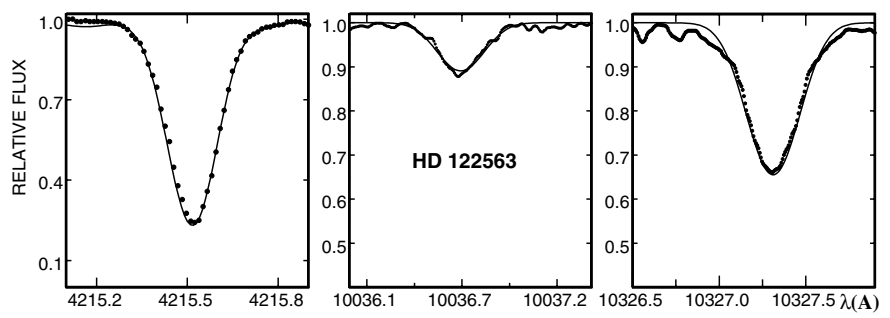

Fig. 3. The same as Fig. 2 but for HD 122563 spectrum. No LTE profiles are shown.

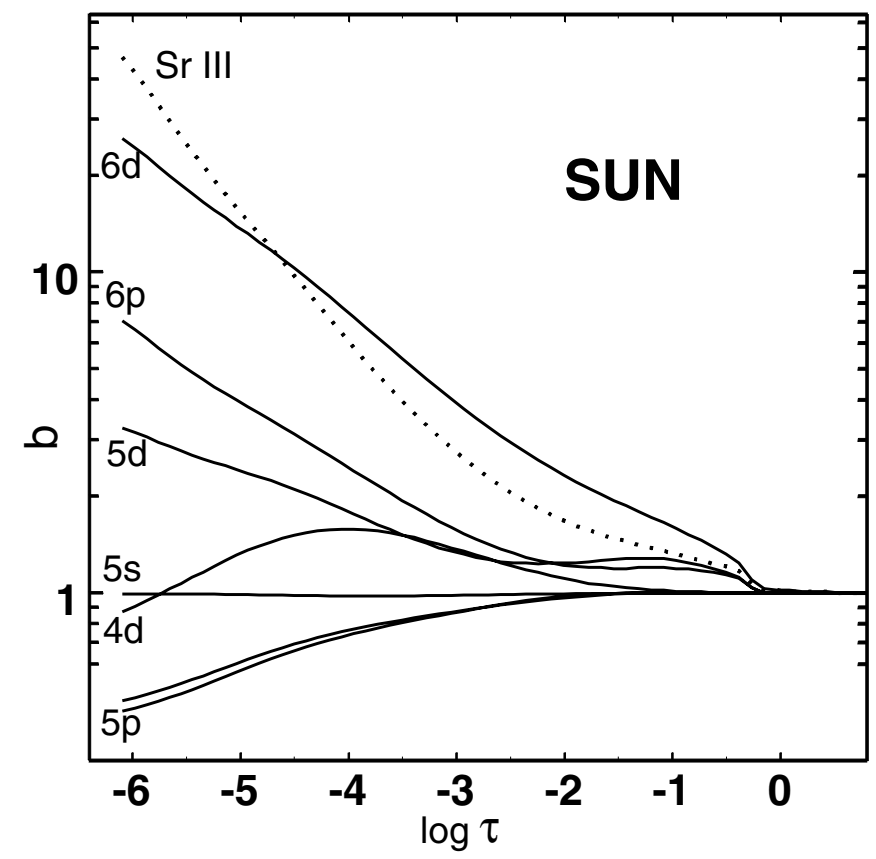

Fig. 4. The distribution of the $b$-factors in the solar atmosphere.

one can note that since $\mathrm{Sr}$ II is a dominating ionization stage of strontium at these temperatures, the population of the ground $5 \mathrm{~s}$ level does not deviate significantly from an equilibrium population. Thus, the resonance lines are stronger, but only slightly because in the range of their formation the $b$-factors of the $5 \mathrm{~s}$ level are close to unity.

For the solar metallicity, the lines $4077 \AA$ and $4215 \AA$ are only slightly affected by the NLTE effects (abundance correction is about 0.01), while for all three IR lines, corrections are rather significant (about 0.3 ).

For the giant stars with metal-deficient atmospheres $\left(\left[\mathrm{Fe} / \mathrm{H}=-3\right.\right.$ and $\left.T_{\text {eff }}=5000 \mathrm{~K}\right)$, the NLTE correction is somewhat different (Fig. 5). At low metallicity, all the lines are formed, on average, in the deeper atmosphere layers. The lower electron concentration leads to significant deviations from LTE in the level populations. The ground level of $\mathrm{Sr}$ II is underpopulated at $\log \tau=-1$, while excited levels are overpopulated. The pumping mechanism increases the population of $5 \mathrm{p}$ level, and consequently the populations of the higher levels become overpopulated too (since $4 \mathrm{~d}$ level is more tightly connected to $5 \mathrm{p}$ than to $5 \mathrm{~s}$, it is also overpopulated).

For the infrared lines, the $b$-factor of the higher level is smaller than that of the lower level and the $b$-factor of the lower level is larger than unity. Altogether, this means that IR lines tend to be stronger. The corresponding NLTE corrections are about

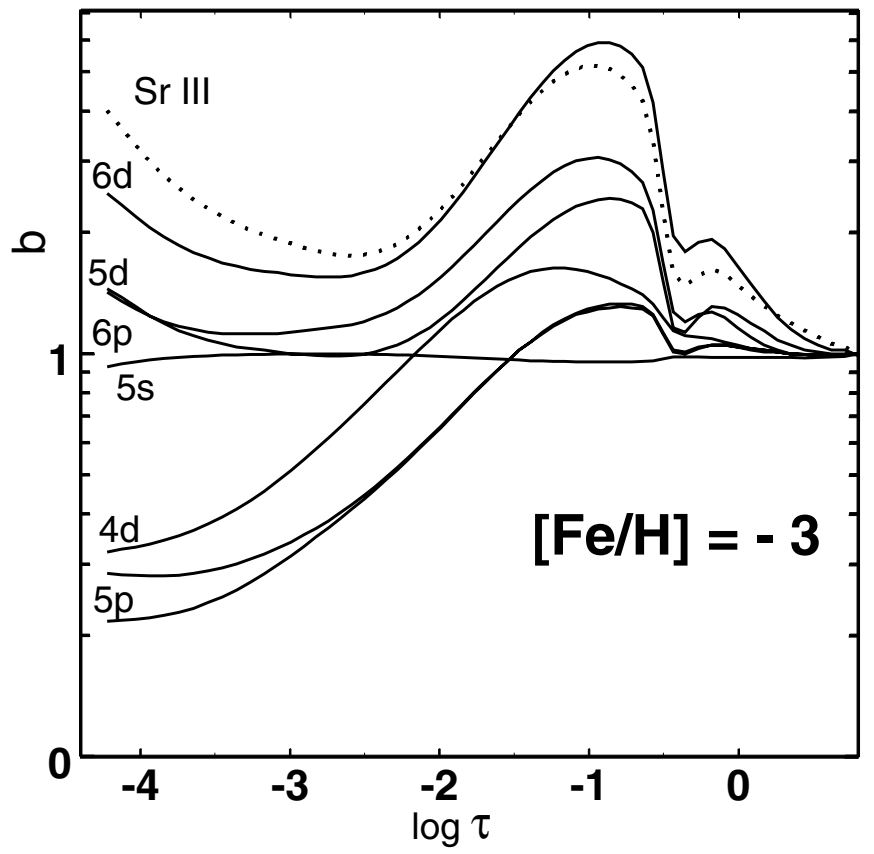

Fig. 5. The same as Fig. 4 but for the metal-poor atmosphere.

-0.5 dex and even larger depending on the metallicity of the atmosphere and other parameters.

The behaviour of the resonance lines were first described in detail by Belyakova \& Mashonkina (1997), and later also considered by Short \& Hauschildt (2006). Here we mention only two cases, which are graphically presented in Fig. 6. At the temperature of $6500 \mathrm{~K}$ and $[\mathrm{Fe} / \mathrm{H}]$ lower than -2 , these lines becomes weaker than those for LTE, and this effect progressively increases with decreasing model metallicity. This behavior reflects that at this temperature and metallicity $\mathrm{Sr}$ II is no longer a completely dominant ionization stage (only up to $70 \%$ atoms persist in the form of a singly ionized strontium), and the ground level of Sr II appears to be significantly underpopulated. For the lower temperatures, the ratio of Sr III to Sr II is about 0.01, and the ground level tends to have an equilibrium population. The change in the profile between NLTE and LTE is small, while the equivalent width is practically the same for both LTE and NLTE. This is because the line core is formed in the upper atmosphere layers where the $b$-factor for $5 \mathrm{p}$ level is much smaller than for 5s (NLTE profile is deeper than LTE), while the far wings are formed at the depth where the situation is opposite. This is a result of the difference in the flux redistribution between the line core and wings. Since in reality the far wings of the line are ignored, the direct use of the equivalent width value in NLTE analysis often produces not quite correct results. In the plot at the right in Fig. 6, we show the LTE and NLTE profiles of the line that comprise almost equal equivalent widths (as follows from the calculations), while the line core regions look different. Therefore, the correct means of deriving NLTE strontium abundance should be individual profile fitting for each star using a complete NLTE computation.

In Fig. 7, we show the dependence of NLTE-LTE abundance correction upon effective temperature and other parameters. We note that NLTE corrections of the strontium abundance depend on the effective temperature and gravity of the model, as well as the strontium abundance itself. 

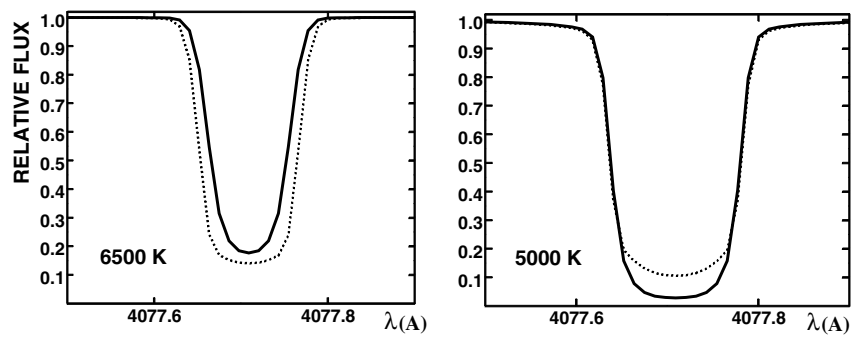

Fig. 6. LTE (dotted line) versus NLTE (continuous line) profiles for the two temperature values.
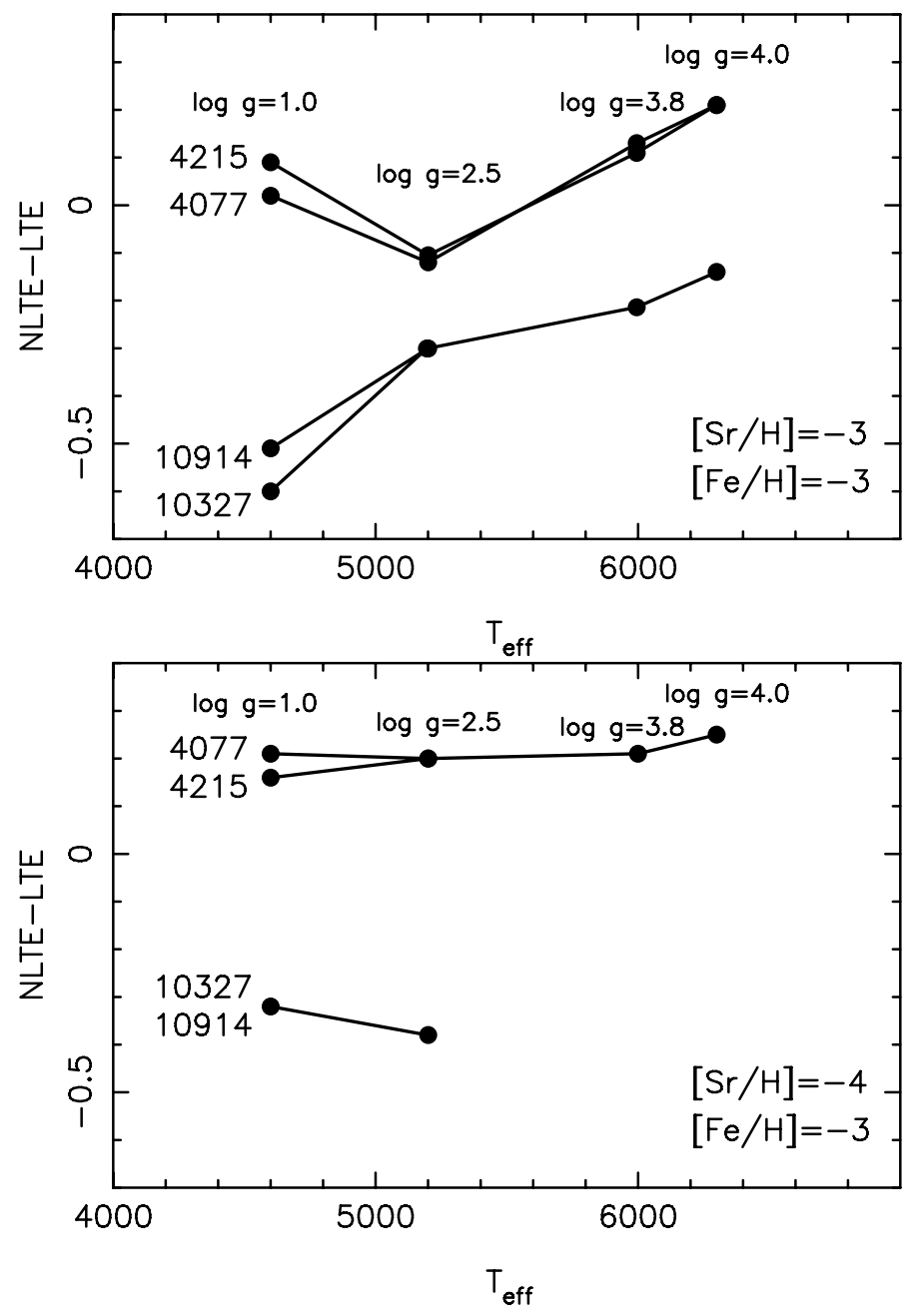

Fig. 7. NLTE correction as a function of $T_{\text {eff }}$ for the different parameters.

\section{NLTE strontium abundance determination in programme stars}

The NLTE abundances of strontium derived in our EMP stars are given in Table 1, and graphically displayed in Fig. 8. The NLTE barium abundances are also provided in the table (when available); they were computed with overshooting Kurucz models (Andrievsky et al. 2009) and are, to ensure homogeneity with Sr abundances, shifted by -0.03 dex for the turn-off stars and -0.05 dex for the giants.

The $\mathrm{Sr}$ and $\mathrm{Ba}$ abundance results shown in the following diagrams are all NLTE abundances.

This work confirms (with some improvements) the main trends previously found for the $\mathrm{Sr}$ abundances, determined

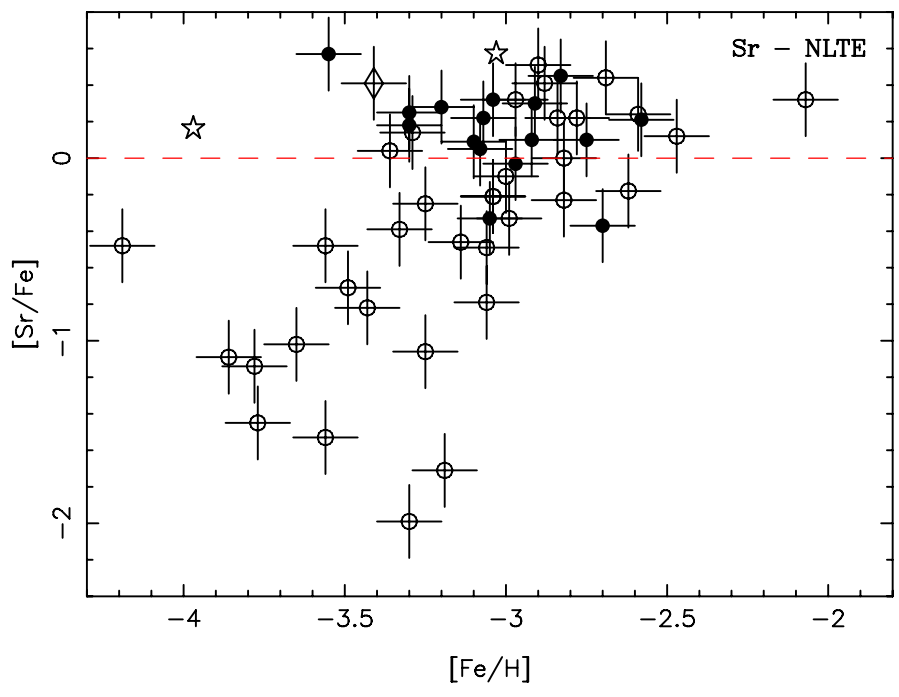

Fig. 8. $[\mathrm{Sr} / \mathrm{Fe}]$ vs. $[\mathrm{Fe} / \mathrm{H}]$ in our program stars: dwarfs: closed circles, giants: open circles, the C-rich stars CS 22949-037 and CS 22892-052: asterisks, the Ba-poor star CS 22897-008: diamond. Three newly analyzed giants are added. Typical error bars have been indicated.

assuming LTE, especially those for the same sample of EMP stars: Fig. 8, is directly comparable to Fig. 12 (upper panel) of Bonifacio et al. (2009). A comparison of the figures (and of the tables) indicates that the NLTE corrections are moderate, but not negligible. The NLTE strontium abundances are, on average, about 0.2 dex higher than the LTE abundances, with variations from star to star.

It has long been known that the scatter in the $\mathrm{Sr}$ abundance is very large (e.g. McWilliam 1998). The scatter in the [Sr/Fe] ratios is slightly smaller when NLTE (rather than LTE) values are used, but even using the NLTE values, this scatter remains very large (Fig. 8). The scatter is also larger at low metallicities (e.g.: McWilliam 1998; François et al. 2007; Bonifacio et al. 2009; Lai et al. 2008): this trend seems to be confirmed in NLTE (Fig. 8), although we note that this conclusion depends on the position of the C-rich star CS 22949-037; since this is also an $\alpha$-elementrich star and therefore does not have a very low metallicity (Aoki et al. 2005), its extremely low $[\mathrm{Fe} / \mathrm{H}]$ value might not be representative.

Despite the large scatter, the abundances of the EMP stars show that, as the iron abundance increases, the $\mathrm{Sr}$ abundance increases, in the mean, far more than $\mathrm{Fe}$, the $[\mathrm{Sr} / \mathrm{Fe}]$ ratio increasing rapidly at first (faster than a secondary process) and stabilizing at about the solar $[\mathrm{Sr} / \mathrm{Fe}]$ ratio after some overshoot. The additional productions of $\mathrm{Sr}$ and $\mathrm{Fe}$ then remain at this constant ratio, up to metallicities of around about $[\mathrm{Fe} / \mathrm{H}]=-1.5$, and the scatter decreases (see François et al. 2007).

The large enhancement of Sr, already detected for the LTE measurements (McWilliam 1998; François et al. 2007) in the (Ba-poor) star CS 22897-008 (diamond at left in Fig. 8) is confirmed by our NLTE determinations. This enhancement is larger than for any of those listed previously in "Sr-rich metal-poor stars" (Truran et al. 2002). The star is intermediate between the Sr-rich stars HD 122563 and the (even richer) star BS 16550087 (Lai et al. 2008), but it has a lower metallicity (and a lower Ba abundance) than the two Sr-rich stars. It could be, as well as HD 122563, considered as an example of a so-called "weak" r-process (Honda et al. 2007): its Eu abundance is too low to be measured but, by that very fact, could be compatible with such a "weak" r-process. 


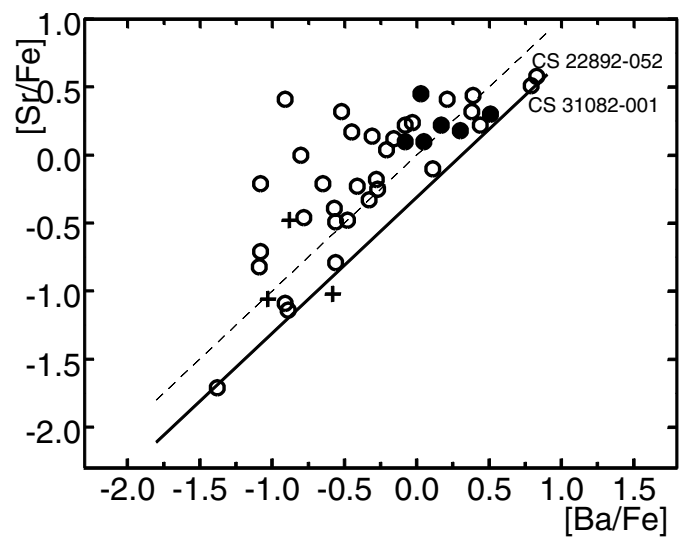

Fig. 9. $[\mathrm{Sr} / \mathrm{Fe}]$ vs. $[\mathrm{Ba} / \mathrm{Fe}]$. The solar ratio $[\mathrm{Sr} / \mathrm{Ba}]=0$ is represented by the dashed line. The value $[\mathrm{Sr} / \mathrm{Ba}]=-0.31$, corresponding to the main r-process production, is represented by the full line. Closed circles: dwarfs, open circles: giants, crosses: three added EMP giants. The stars without Ba measurements are not represented. Symbols as in Fig. 8. In Fig. 9 and in following figures, the error bars are no longer indicated for an easier visual perception of the abundance trends.

The low metallicity turn-off star CS 29527-015 (extreme left closed circle in Fig. 8) is Sr-rich, its $\mathrm{Ba}$ and $\mathrm{Eu}$ abundances being too low to be measured.

\subsection{Srvs. Ba}

Figure 9 shows $[\mathrm{Sr} / \mathrm{Fe}]$ versus $[\mathrm{Ba} / \mathrm{Fe}]$. Both $\mathrm{Sr}$ and Ba globally increase with $\mathrm{Fe}$. Since the $\mathrm{Sr} / \mathrm{Fe}$ ratio has a larger scatter at low metallicities, it also has more scatter at low values of $[\mathrm{Ba} / \mathrm{Fe}]$.

In this figure, the thick line corresponds to the constant ratio $[\mathrm{Sr} / \mathrm{Ba}]=-0.31$, which is the value chosen by Qian $\&$ Wasserburg (2008) to represent their component for the Sr-poor stars. This ratio is compatible with the production ratio of $\mathrm{Sr}$ and $\mathrm{Ba}$ for the main r-process: it is near (but very slightly smaller than) the ratio given by Burris et al. (2000) for the r-processonly component of these elements in the Solar system, it is also slightly below the similar value provided for this component by Arlandini et al. (1999).

In Fig. 9, all the stars (apart from one, marginally) are situated higher than the line suggesting that, for most stars, one (or more) additional production(s) of Sr occurred (or a variation in the main r-process, or both). The departures from the main r-process are higher at low metallicities (and at low Ba abundances).

In our sample, two r-rich stars (CS 31082-001, CS 22892052) defining the main r-process (Cowan $\&$ Sneden, 2006), are (as expected) near the solid line (and also BD $+17^{\circ} 3248$ ). In their survey of r-rich stars (r-II stars), Barklem et al. (2005) note that the r-II stars are centred on a metallicity of $[\mathrm{Fe} / \mathrm{H}]=-2.81$, and the two r-rich giants in our sample, have indeed such relatively high metallicities (and are consequently relatively $\mathrm{Sr}$ and $\mathrm{Ba}-$ rich, in the figure being near the upper corner). The Sr-rich turnoff star (closed circle at left in Fig. 8) has a significanly lower metallicity.

In Fig. 9, it is seen that the upper envelope has a $[\mathrm{Sr} / \mathrm{Fe}]$ ratio that is approximately constant, while $[\mathrm{Ba} / \mathrm{Fe}]$ increases by an order of magnitude, suggesting that the production process of $\mathrm{Sr}$ is different (somehow independent?) of the process producing $\mathrm{Ba}$. With increasing $\mathrm{Fe},[\mathrm{Ba} / \mathrm{Fe}]$ increases thanks to the main r-process, while along the upper envelope $[\mathrm{Sr} / \mathrm{Fe}]$ does not. This

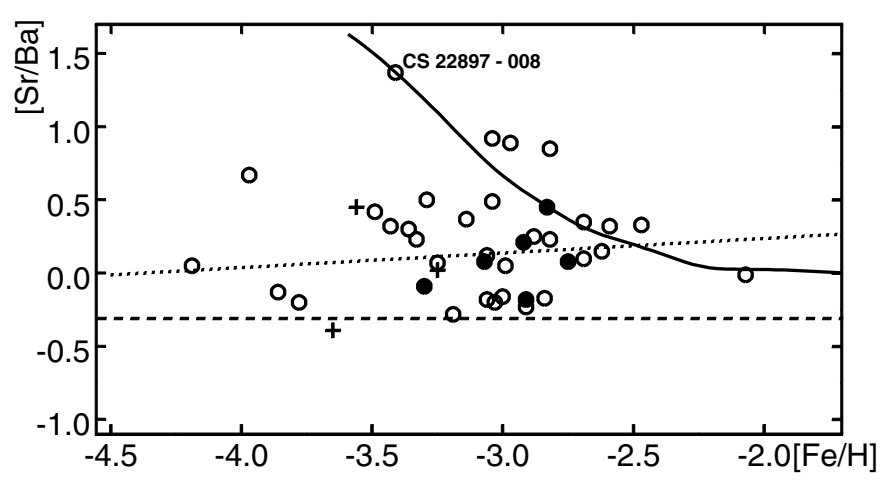

Fig. 10. [Sr/Ba] vs. [Fe/H]. LEPP model prediction represented by the thick line, $[\mathrm{Sr} / \mathrm{Ba}]=-0.31$ : dashed line, linear regression: dotted line.

at least indicates that an early production of Sr occurred in the chemical evolution of the Galaxy.

Figure 10 shows the positions of the stars in the $[\mathrm{Sr} / \mathrm{Ba}]$ vs. $[\mathrm{Fe} / \mathrm{H}]$ plot, together with a plot of the theoretical LEPP model predictions (Travaglio et al. 2004). The corresponding theoretical curve is not far from the upper envelope of the data points, and an inhomogeneous mixing of the products of such a LEPP process with the products of the main r-process could explain the distribution of the stars in this figure.

In our sample, the carbon-rich EMP star CS 22949-037 is classified as a "CEMP-no" star: this classification is explained e.g. by Sivarani et al. (2006). This carbon-rich star with the rather high ratio $[\mathrm{Sr} / \mathrm{Ba}] \approx 0.6$ could also be explained by the LEPP process (or by massive rotating stars e.g., Pignatari et al. 2008; Meynet et al. 2010, or other processes; see discussion below).

\subsection{Production of $\mathrm{Fe}, \mathrm{Sr}$, and $\mathrm{Ba}$}

François et al. (2007) demonstrated (their Fig. 15) that in extremely metal-poor stars, the ratio $[\mathrm{Sr} / \mathrm{Ba}]$ (as well as $\mathrm{Y} / \mathrm{Ba}$ and $\mathrm{Zr} / \mathrm{Ba}$ ) is often higher than solar in the range $-3.7>[\mathrm{Ba} / \mathrm{H}]>$ -4.7. A correlation is found between $[\mathrm{Sr} / \mathrm{Ba}]$ and $[\mathrm{Ba} / \mathrm{H}]$ in the interval $-5.5<[\mathrm{Ba} / \mathrm{H}]<-4.5$ (LTE values). At very low metallicity, François et al. (2007) find that, for the lower observed values of $[\mathrm{Ba} / \mathrm{H}]$ (about $-5 \mathrm{dex})$, the ratio $[\mathrm{Sr} / \mathrm{Ba}]$ returns to the solar values. Our NLTE homogeneous determinations qualitatively confirm this behaviour, and enable one to robustly claim that the $\mathrm{Sr}$ abundances are generally higher than those predicted by the main r-process pattern.

Figure 11 shows that, with $[\mathrm{Ba} / \mathrm{H}]$ increasing from -5.0 to -4.0 , the upper envelope of the scatter diagram reaches the one to one slope when excluding the star CS 22897-008 (and even more when including this star). Along this envelope, the Sr abundance increases much more than the $\mathrm{Ba}$ abundance. In the other part of the figure, where $[\mathrm{Ba} / \mathrm{H}]$ increases from -3.5 to -2.5 , the upper envelope decreases with a slope around -0.7 ; the $\mathrm{Sr}$ abundance increases more gradually than $\mathrm{Ba}$. This behaviour illustrates the well-known lag between the onsets of $\mathrm{Sr}$ and $\mathrm{Ba}$ production, again suggesting that the production processes of $\mathrm{Sr}$ and $\mathrm{Ba}$ are different.

Many stars are relatively near the dashed line (corresponding to the main r-process), about half of the stars having $\mathrm{Sr} / \mathrm{Ba}$ ratios smaller than three times the main r-process ratio.

The three stars with the lowest $\mathrm{Ba}$ abundances (including $\mathrm{CD}-38^{\circ} 245$ ) have $\mathrm{Sr} / \mathrm{Ba}$ ratios near the main r-process ratio (in 


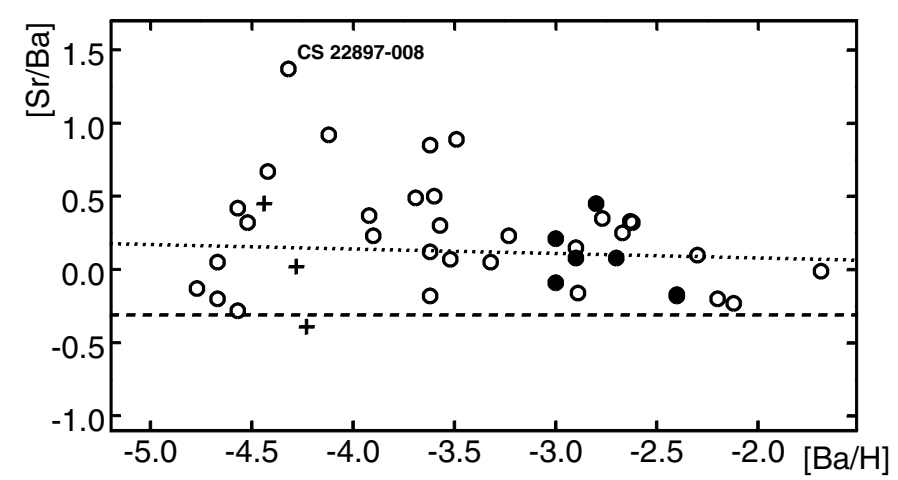

Fig. 11. $[\mathrm{Sr} / \mathrm{Ba}]$ vs. $[\mathrm{Ba} / \mathrm{H}]$. $[\mathrm{Sr} / \mathrm{Ba}]_{H}=-0.31-$ dashed line, linear regression - dotted line.

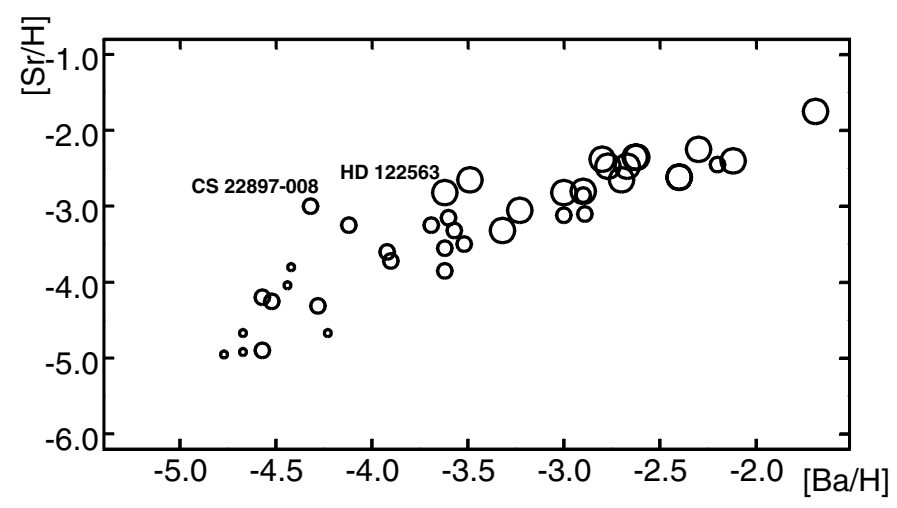

Fig. 12. $[\mathrm{Sr} / \mathrm{H}]$ vs. $[\mathrm{Ba} / \mathrm{H}]$. Stars with $[\mathrm{Fe} / \mathrm{H}]<-3.5-$ small circles, $-3.5<[\mathrm{Fe} / \mathrm{H}]<-3.0$-intermediate circles, $[\mathrm{Fe} / \mathrm{H}]>-3.0-$ large circles.

agreement with the behaviour of the most Ba deficient star of Lai et al. (2008).

Figure 12 shows the simultaneous enrichment of $\mathrm{Fe}, \mathrm{Sr}$, and $\mathrm{Ba}$, the low metallicity stars being both Ba-poor and Sr-poor. Figure 12 is somewhat similar to Figure 9 but provides an indication of the iron production.

Summarizing, we can state that our NLTE Sr and Ba abundances discussed above appear to be consistent with the Qian \& Wasserburg (2008) simple three-component model of chemical evolution. According to that model, the first phase of the chemical evolution of the Galaxy would be the production of elements by high-energy hypernovae: their yields would include significant amount of low-A elements (from $\mathrm{Na}$ to $\mathrm{Zn}$, including iron), but no heavier species. In reality, Fig. 12 shows that ironpoor stars have a very low content of $\mathrm{Sr}$ and $\mathrm{Ba}$. The shortfall in Sr later decreases to zero when the low-mass and normal-mass $\mathrm{SNe}$ ( $H$ and $L$ sources respectively) begin producing strontium (Fig. 8). Moreover, since $\mathrm{Sr}$ is produced by both the $H$ and $L$ sources, and $\mathrm{Ba}$ is produced predominantly by the lower-mass $H$ source, it can explain the general decrease in the $\mathrm{Sr} / \mathrm{Ba}$ scatter, which is clearly seen seen in Fig. 11.

\subsection{Comparison to faint dwarf spheroidal galaxies}

We have much to learn about dSphs, but a full discussion is beyond the scope of this paper. We present only two short comparisons.
Cohen \& Huang (2010) find, in the (metal-poor) stars of the Ursa Minor dwarf spheroidal galaxy, the heavy elements produced follow the main r-process pattern: this is consistent with the short duration of the star-forming phase of this small galaxy, inferred from the CMDs (Orban et al. 2008).

Norris et al. (2010) identify CEMP-no stars (or similar) in dwarf galaxies, and note a similarity between their abundance patterns and those of the same stars in our Galaxy: the similarity is indeed striking, but strontium is systematically stronger in the stars of our Galaxy. The comparison is made for LTE abundances, but for one of them (CS 22949-037), the high Sr abundance is confirmed by our NLTE determinations.

\subsection{Sr formed by other processes?}

Many independent explanations of excess of Sr production relative to the main r-process have been proposed, such as

1) a quenched flux model (Truran et al. 2002);

2) particular models of hypernovae (e.g. special computation of four models of hypernovae) reproducing the high $\mathrm{Sr} / \mathrm{Ba}$ ratios (and other abundances) in four stars of our sample, by Izutani et al. (2009);

3 ) the inhomogenous mixing of three types of nucleosynthesis productions: a phenomenological "three-component model" (Qian \& Wasserburg 2008);

4) a weak r-process (Honda et al. 2007) or a variation in the r-process (Roederer et al. 2010);

5) contamination by main s-process (by an AGB companion): metal-poor AGB do not generally provide a strong $\mathrm{Sr}$ excess, still, monitoring a subsample of Sr-rich stars would be useful;

6) a LEPP process (Travaglio et al. 2004).

Since nitrogen is observed as a primary product in some EMP stars (Spite et al. 2005), rotating massive stars may produce heavy elements, and in some cases strong $\mathrm{Sr} / \mathrm{Ba}$ ratios (Pignatari et al. 2008). However, the correlation of $\mathrm{Sr} / \mathrm{Ba}$ with the abundance of $\mathrm{N}$ is weak, suggesting that, if rotating stars are a source of $\mathrm{Sr}$, it is not the unique additional source. The Sr-rich star CS 22949-037, classified as CEMP-no, might be produced by a LEPP, or by a massive rotating star (producing also carbon).

\section{Conclusion}

The homogeneous NLTE Sr abundances of the stars of this sample, provide precise information about the $\mathrm{Sr}$ abundances in the early evolution of the Galaxy. In addition to previously determined NLTE Ba abundances, these data provide some insight into the process(es) of Sr production.

The methods of the NLTE determination of Sr abundances have been verified (solar spectrum, extension in infrared), the results being in satisfactory agreement with computations found in the literature (e.g. Belyakova \& Mashonkina 1997; Mashonkina et al. 2008) and comparisons with a few stellar NLTE abundances available in the literature show good agreement.

Our study here has confirmed the behaviour of the Sr LTE abundances found in the literature for both metal-poor stars (e.g. by McWilliam 1998; and Lai et al. 2008) and our sample (François et al. 2007; and Bonifacio et al. 2009). These behaviours are now established on a firmer basis.

During the chemical evolution of the Galaxy, when the iron abundance increases, the mean $\mathrm{Sr}$ abundance increases, the mean $[\mathrm{Sr} / \mathrm{Fe}]$ ratio increases, reaching (and then exceeding) the solar ratio, before decreasing slowly towards the solar ratio. 
The $\mathrm{Sr} / \mathrm{Ba}$ ratio is always higher than the main r-process ratio. In addition to the large scatter in the $\mathrm{Sr} / \mathrm{Ba}$ ratios, this suggests that either some production of $\mathrm{Sr}$ (at least partly independent of the production of $\mathrm{Ba}$ ) is added to the main r-process, or that the r-process that produces $\mathrm{Sr}$ and $\mathrm{Ba}$ varies significantly.

A number of additional processes of $\mathrm{Sr}$ enrichment have been proposed in the literature, although none have been clearly verified, the data even being consistent with several processes existing simultaneously, enhancing the difficulty of identifying these processes.

In this complex situation, it is of the upmost importance to collect new accurate abundances for a larger sample of stars, and a larger number of heavy elements (first and second peak elements) in the metallicity range $-4.5<[\mathrm{Fe} / \mathrm{H}]<-2.5$, to try to identify the processes (and their sites) responsible for producing these elements.

Acknowledgements. Authors would like to thank Andrea Dupree for sending the Keck IR spectra of two stars: HD 1581 and HD 122563. This work made use of the CDS, ADS and VALData-base. S.M.A. and S.A.K. are thankful to GEPI for the hospitality during the stay in Paris-Meudon Observatoire, and would like to acknowledge partial support from SCOPES grant No. IZ73Z0-128180/1 and from the contract UKR CDIV N24008 in the France (CNRS) - Ukraine (National Academy of Sciences) exchange programmme. F.S. and M.S. acknowledge partial support from PNPS (CNRS). We thank the anonymous referee for valuable comments that improved the paper, and language editor Claire Halliday for her help.

\section{References}

Allen, C. W. 1973, Astrophysical Quantities (London: Athlone Press) Alonso, A., Arribas, S., \& Martínez-Roger, C. 1999, A\&AS, 140, 261 Andrievsky, S. M., Spite, M., Korotin, S. A., et al. 2009, A\&A, 494, 1083 Aoki, W., Honda, S., Beers, T. C., et al. 2005, ApJ, 632, 611

Arlandini, C., Käppeler, F., Wisshak, K., et al. 1999, ApJ, 525, 886

Asplund, M., Grevesse, N., \& Sauval, A. J. 2005, in ASP Conf. Ser., 336, 25

Bagnulo, S., Jehin, E., Ledoux, C., et al. 2003, The ESO Paranal Science Operations Team, Messenger, 114, 10

Ballester, P., Modigliani, A., Boitquin, O., et al. 2000, Msngr, 101, 31

Barklem, P. S., Christlieb, N., Beers, T., et al. 2005, A\&A, 439, 129

Belyakova, E. V., \& Mashonkina, L. I. 1997, ARep, 41, 530

Bonifacio, P., Molaro, P., Sivarani, T., et al. 2007, A\&A, 462, 851 ("First Stars VII")

Bonifacio, P., Spite, M., Cayrel, R., et al. 2009, A\&A, 501, 519 ("First Stars XII")

Borsenberger, J., Praderie, F., \& Michaud, G. 1981, ApJ, 243, 533

Burris, D. L., Pilachowski, C. A., Armandroff, T. E., et al. 2000, ApJ, 544, 302

Carlsson, M. 1986, Uppsala Obs. Rep., 33

Castelli, F., Gratton, R. G., \& Kurucz, R. L. 1997, A\&A, 318, 841

Cayrel, R., Depagne, E., Spite, M., et al. 2004, A\&A, 416, 1117 ("First Stars V")

Cohen, J. G., \& Huang, W. 2010, ApJ, 719, 931
Cowan, J. J., \& Sneden, C. 2006, Nature, 440, 1151

Cowan, J. J., \& Thielemann, F.-K. 2004, Phys. Today, 57, 47

Dekker, H., D’Odorico, S., Kaufer, A., Delabre, B., \& Kotzlowski, H. 2000 Optical and IR Telescope Instrumentation and Detectors, ed. I. Masanori , \& A. F. Moorwood, Proc. SPIE, 4008, 534

Drawin, H.-W. 1961, ZPh, 164, 513

Farouqi, K., Kratz, K.-L., Mashonkina, L. I., et al. 2009, ApJ, 694, L49

Fischer, T., Whitehouse, S. C., Mezzacappa, A., Thielemann, F.-K., \& Liebendoerfer, M. 2010, A\&A, 517, A80

François, P., Depagne, E., Hill, V., et al. 2007, A\&A, 476, 935 ("First Stars VIII") Goldsmith, S., \& Boxman, R. L. 1981, J. Phys. B, 14, 3031

Hoffman, R. D., Mueller, B., \& Janka, H.-T. 1997, ApJ, 482, 951

Hoffman, R. D., Mueller, B., \& Janka, H.-T. 2008, ApJ, 676, L127

Honda, S., Aoki, W., Ishimaru, Y., \& Wanajo, S. 2007, ApJ, 666, 1189

Hüdepohl, L., Müller, B., Janka, H.-Th., Marek, A., \& Raffelt, G. 2010, Phys Rev. Lett., 104, 1101

Izutani, N., Umeda, H., \& Tominaga, N. 2009, ApJ, 692, 1517

Korotin, S. A., Andrievsky, S. M., \& Luck, R. E. 1999, A\&A, 351, 168

Kurucz, R. 1993, ATLAS9 Stellar Atmosphere Programs and $2 \mathrm{~km} / \mathrm{s}$ grid. Kurucz CD-ROM No. 13, Cambridge, Mass.: Smithsonian Astrophysical Observatory

Kurucz, R. L. 2005, Mem. Soc. Astron. Ital. Suppl., 8, 14

Kurucz, R. L., Furenlid, I., Brault, J., \& Testerman, L. 1984, Solar flux atlas from 296 to $1300 \mathrm{~nm}$

Lai, D., Bolte, M., Johnson, J. A., et al. 2008, ApJ, 681, 1524

Lang, K. 1998, Astrophysical formulae (Berlin - Heidelberg - NewYork: Springer-Verlag)

Lingard, A., \& Nielsen, S. E. 1977, ADNDT, 19, 532

Mashonkina, L. I., \& Gehren, T. 2001, A\&A, 376, 232

Mashonkina, L., Zhao, G., Gehren, T., et al. 2008, A\&A, 478, 529

McWilliam, A. 1998, AJ, 115, 1640

Meynet, G., Hirschi, R., Ekström, S., et al. 2010, A\&A, 521, A30

Norris, J. E., Gilmore, J., Wyse, R. F. G., et al., 2010, ApJ, 722, L104

Orban, C., Gnedin, O. Y., Weisz, D. R., et al. 2008, AJ, 686, 1030

Peach, G. 1967, Mem. RAS, 71, 13

Pignatari, M., Gallino, R., Meynet, G., et al. 2008, ApJ, 687, L95

Qian, Y. Z., \& Wasseburg, G. J. 2008, ApJ, 687, 272

Roberts, L., Woosley, S., \& Hoffman, D. 2010, ApJ, 722, 954

Roederer, I. U., Cowan, J. J., Karakas, A. I., et al. 2010, ApJ, 724, 975

Short, C. I., \& Hauschildt, P. H. 2006, ApJ, 641, 494

Sivarani, T., Beers T. C., Bonifacio, P., et al. 2006, A\&A, 459, 125

Smith, G. H., Dupree, A. K., \& Strader, J. 2004, PASP, 116, 819

Sobelman, I. I., Vainshtein, L. A., \& Yukov, E. 1981, Excitation of Atoms and Broadening of Spectral Lines, Springer Ser. in Chem. Phys. (Berlin: Springer)

Spite, M., Cayrel, R., Plez, B., et al. 2005, A\&A, 430, 655 ("First stars VI")

Steenbock, W., \& Holweger, H. 1992, LNP, 401, 57

Travaglio, C., Gallino, R., Arnone, E., et al. 2004, ApJ, 601, 864

Truran, J. W., Cowan, J. J., Pilachowski, C. A., \& Sneden, C. 2002, PASP, 114, 1293

Tsymbal, V. V. 1996, Model Atmospheres and Spectrum Synthesis, ed. S. J. Adelman, F. Kupka, \& W. W. Weiss, San Francisco, ASP Conf. Ser., 108 van Regemorter H. 1962, ApJ, 136, 906

Wanajo, S., \& Janka, H.-J. 2010, in The 10th international symposium on origin of matter and evolution of galaxies: OMEG-2010, AIP Conf. Proc., 1269, 120 Warner, B. 1968, MNRAS, 139, 115

Wiese, W. L., \& Martin, G. A. 1980, NSRDS-NBS 68 (Part II) 